\title{
"Smart" nickel oxide based core-shell nanoparticles for combined chemo and photodynamic cancer therapy
}

\author{
Shazia Banol-3,* \\ Samina Nazir ${ }^{2, *}$ \\ Saeeda Munir ${ }^{3}$ \\ Mohamed Fahad AlAjmi ${ }^{4}$ \\ Muhammad Afzal' \\ Kehkashan Mazhar ${ }^{3}$ \\ 'Department of Physics, The \\ Islamia University of Bahawalpur, \\ ${ }^{2}$ Nanosciences and Technology \\ Department, National Centre for \\ Physics, Islamabad, ${ }^{3}$ Institute of \\ Biomedical and Genetic Engineering, \\ Islamabad, Pakistan; ${ }^{4}$ College of \\ Pharmacy, King Saud University, \\ Riyadh, Kingdom of Saudi Arabia \\ *These authors contributed equally \\ to this work
}

This article was published in the following Dove Press journal:

International Journal of Nanomedicine

12 July 2016

Number of times this article has been viewed

\begin{abstract}
We report "smart" nickel oxide nanoparticles (NOPs) as multimodal cancer therapy agent. Water-dispersible and light-sensitive $\mathrm{NiO}$ core was synthesized with folic acid (FA) connected bovine serum albumin (BSA) shell on entrapped doxorubicin (DOX). The entrapped drug from NOP-DOX@BSA-FA was released in a sustained way (64 hours, $\mathrm{pH}=5.5$, dark conditions) while a robust release was found under red light exposure (in $1 / 2$ hour under $\lambda_{\text {max }}=655 \mathrm{~nm}$, $50 \mathrm{~mW} / \mathrm{cm}^{2}$, at $\mathrm{pH}=5.5$ ). The cell viability, thiobarbituric acid reactive substances and diphenylisobenzofuran assays conducted under light and dark conditions revealed a high photodynamic therapy potential of our construct. Furthermore, we found that the combined effect of DOX and NOPs from NOP-DOX@BSA-FA resulted in cell death approximately eightfold high compared to free DOX. We propose that NOP-DOX@BSA-FA is a potential photodynamic therapy agent and a collective drug delivery system for the systemic administration of cancer chemotherapeutics resulting in combination therapy.
\end{abstract}

Keywords: light-triggered drug release, cancer, bovine serum albumin, multi-model therapy

\section{Introduction}

Delivery of large amounts of cancer therapeutics intended for sustained and triggered release still faces significant challenges. Strategies to develop cancer cellspecific therapeutics vary, but all attempts use nanoscaled carriers such as dendritic macromolecules, ${ }^{1-3}$ liposomes, ${ }^{4}$ polymers,${ }^{5}$ or metal nanoparticles ${ }^{6}$ to transport and target therapeutic agents. The desired result is more effective tumoricidal activity ${ }^{7}$ and lesser toxicity in normal cells. A newer advancement is the combination cancer therapy technique, which is used for invading cancers through more than one mechanism, such as a combination of targeted drug delivery and photodynamic therapy (PDT) or photothermal therapy. Combined treatments may target different key signal transduction pathways in order to amplify overall therapeutic efficacy. ${ }^{8,9}$ This may result in synergistic effects, so that a reduction of the drug dose becomes feasible, may eliminate or at least reduce the side effects usually caused by chemotherapeutic agents such as doxorubicin (DOX). ${ }^{10,11}$ A progressively emerging system in such efforts is the lighttriggered release of chemotherapeutics from light-responsive nanocarriers; capable enough to damage or destroy malignant tissues by absorbing external electromagnetic energy. ${ }^{8-11}$ Thus, the combination of light and chemotherapeutic agents is a predominantly encouraging methodology for optimizing cancer treatment. Such light-triggered drug release can easily be combined with the photodynamic or photothermal modes, ensuring enhanced cancer cell killing through combination therapy.
Correspondence: Samina Nazir Nanosciences and Technology Department, National Centre for Physics, Islamabad 44000, Pakistan

Tel +923028505080

Email seegasami0I@yahoo.com (c) (1) (5) 2016 Bano et al. This work is published and licensed by Dove Medical Press Limited. The full terms of this license are available at https://www.dovepress.com/terms.php
and incorporate the Creative Commons Attribution - Non Commercial (unported, v3.0) License (http://creativecommons.org/licenses/by-nc/3.0/). By accessing the work you hereby accept the Terms. Non-commercial uses of the work are permitted without any further permission from Dove Medical Press Limited, provided the work is properly attributed. For permission for commercial use of this work, please see paragraphs 4.2 and 5 of our Terms (https://www.dovepress.com/terms.php). 
Here we report an impressively assimilated nickel oxide nanoparticle (NOP)-based conjugate termed NOP-DOX@, bovine serum albumin (BSA)-folic acid (FA) for cumulative drug delivery and in vitro PDT on human cervical epithelial malignant carcinoma (HeLa) cells.

ForNOP-DOX@BSA-FA, we loaded red light-responsive, water-dispersible $\mathrm{NiO}$ nanocrystals with DOX and developed an FA connected BSA soft shell on it. Red light-responsive NOP-DOX@BSA-FA was believed to enhance cancer cell death through the concurrent release of chemotherapeutic drugs from the BSA shell and reactive oxygen species (ROS) produced from the $\mathrm{NiO}$ core in in vitro conditions. This drug carrier system is adept at light-triggered drug release, giving an $\sim 100 \%$ release enhancement compared to the dark conditions besides a desired $\mathrm{pH}$-sensitive release for tumor targeting. This makes NOP-DOX@BSA-FA a reliable choice as an efficient drug delivery system.

\section{Materials and methods Chemicals}

Nickel (II) acetylacetonate (95\%), oleylamine, oleic acid, sodium citrate, $\mathrm{DOX} \cdot \mathrm{HCl}(98.0 \%)$, bovine serum albumin, glutaraldehyde, folic acid (98\%), dimethyl sulfoxide (DMSO, 99\%), $N$-hydroxy succinimide (NHS, 98\%), 1-ethyl-3-(3dimethylaminopropyl) carbodiimide hydrochloride (EDAC), sodium azide and mannitol were purchased from SigmaAldrich (USA); 1,3-Diphenylisobenzofuran (DPBF) from Thermo Fisher Scientific (Waltham, MA, USA); methylene blue from Merck and Co. All other chemicals were analytical grade and used directly.

\section{Synthesis of $\mathrm{NiO}$ nanoparticles (NOPs)}

$\mathrm{NiO}$ cores were synthesized via thermolysis, with thermal decomposition of the metal acetylacetonate (II) precursors. Nickel (II) acetylacetonate $(0.2 \mathrm{mmol})$, oleic acid $(5 \mathrm{~mL})$, and oleylamine $(5 \mathrm{~mL})$ were mixed in a $100 \mathrm{~mL}$ two-neck flask. The solution was heated to $120^{\circ} \mathrm{C}$ with vigorous stirring under vacuum for 30 minutes, and then the temperature was increased to $200^{\circ} \mathrm{C}$ under an argon atmosphere and kept at $200^{\circ} \mathrm{C}$ for 60 minutes. The reaction solution was further heated to $300^{\circ} \mathrm{C}$ at a heating rate of $10^{\circ} \mathrm{C} \mathrm{min}^{-1}$ and kept at $300^{\circ} \mathrm{C}$ for 60 minutes under magnetic stirring. After being cooled down to room temperature, the reaction mixture was precipitated by adding isopropanol $(45 \mathrm{~mL})$ and collected by centrifugation (8,000 rpm, 10 minutes).

A solution of NOPs $(1 \mathrm{mg} / 10 \mathrm{~mL})$ in tetrahydrofuran (THF) was added into water $(4 \mathrm{~mL})$ containing sodium citrate $(8 \mathrm{mg})$ and $\mathrm{NaHCO}_{3}(15 \mathrm{mg})$. The mixture was then heated to $95^{\circ} \mathrm{C}$ and magnetically stirred for 4 hours, cooled to room temperature, and centrifuged (7,000 rpm, 10 minutes) to collect the product.

\section{Characterization}

Characterization of the reaction product was carried out spectroscopically. Low-resolution transmission electron micrographs were obtained on a field emission scanning electron microscope (FE-SEM) (TESCAN, Brno, Czech Republic), Mire 3 XMU $1.2 \mathrm{~nm}$, operating at a voltage of $230 \mathrm{~V}$. The samples were carbon-coated prior to the analysis. Clear solutions of samples were prepared in Milli-Q water for TEM mod and dropped onto copper grids covered by an amorphous carbon film.

X-ray diffraction was performed on a D8 Advance X-ray Diffractometer (Bruker Corporation, Billerica, MA, USA) with $\mathrm{CuK} \alpha$ radiations $\left(\lambda=1.54056 \mathrm{~A}^{0}\right)$ at $45 \mathrm{kV}$ energy. The hydrodynamic diameter was measured with Zetasizer Nano ZSPTM (Malvern Instruments Ltd, Malvern, UK). Magnetic measurements were carried out by using a 7,407 vibrating sample magnetometer (Lake Shore Cryongenics, Inc., Westerville, OH, USA).

Ultraviolet-visible (UV-VIS) spectra were recorded in an optical-quality quartz cuvette with a $1 \mathrm{~cm}$ path length of the instrument (LAMBDA ${ }^{\mathrm{TM}} 25 \mathrm{UV}-\mathrm{VIS} /$ near infrared (NIR); PerkinElmer, Waltham, MA, USA) at room temperature $\left(25^{\circ} \mathrm{C}\right)$. The elemental composition of NOPs was evaluated through Rutherford Backscattering Spectroscopy (RBS). Photoluminescence spectra were obtained on an LS 55 Photoluminescence spectrophotometer (PerkinElmer) using a xenon lamp as the excitation source at room temperature. Fourier transform infrared (FTIR) spectra were recorded using a Bruker Tensor 27 ATR ZnSe FTIR spectrometer (Bruker Corporation).

\section{Synthesis of NOP-DOX@BSA}

DOX was loaded by incubation with NOPs at a 3:1 mass ratio in Milli-Q water for 24 hours undisturbed at room temperature. Free DOX was then removed via centrifugation $(6,000 \mathrm{rpm}$, 20 minutes). BSA $2 \%$ solution was added to the NOP-DOX solution and incubated for 4 hours. Ethanol was added drop-wise until the solution became turbid. Glutaraldehyde (6\%) was added drop-wise into the above solution to allow crosslinking of protein. The resulting solution was further dialyzed for 4 hours against Milli-Q water with Spectra Por 6 Spectrum $^{\circledR}$ dialysis membrane (Spectrum Laboratories, Inc., Rancho Dominguez, CA, USA). The molecular weight cutoff [MWCO] was $35 \mathrm{kDa}$.

\section{Synthesis of NOP-DOX@BSA-FA}

FA $(0.25 \mathrm{~g})$ was dissolved in $20 \mathrm{~mL}$ DMSO and the mixture was subjected to sonication for 45 minutes. The carboxylate 
group of FA was activated by the addition of NHS and EDAC. The reaction was allowed to take place under nitrogen flow for 12 hours. Molar ratio of FA/NHS/EDAC was kept as $2: 2: 1$. The resultant mixture was filtered for further use. ${ }^{1}$ The activated FA $(1 \mathrm{~mL})$ was reacted with NOP-DOX@BSA $(9 \mathrm{~mL})$ solution. The mixture was purged with argon with continuous stirring for 4 hours, centrifuged, and the resulting solution of NOP-DOX@BSA-FA was purified against nanopure using membrane tubing (MWCO 3,500) to remove unreacted reagents. The purified complex was analyzed using UV-VIS spectrometry and FTIR.

\section{Drug release from NOP-DOX@BSA-FA}

DOX release from the NOP-DOX@BSA-FA in phosphatebuffered saline ( $\mathrm{pH} 5.4,7.4,9)$ under dark conditions maintained at $37^{\circ} \mathrm{C}$ was evaluated using the dynamic dialysis method.NOP-DOX@BSA-FA suspension $(5 \mathrm{mg} / \mathrm{mL})$ placed in a dialysis tubing (MWCO $35 \mathrm{kDa}$ ) was immersed in a $100 \mathrm{~mL}$ beaker covering $80 \mathrm{~mL}$ release buffer. The beaker was placed in an incubator shaker at $100 \mathrm{rpm}$. The release buffer ( $5 \mathrm{~mL}$ aliquots) was removed from the beaker at regular time intervals. DOX concentration in each sample was quantified by UV spectrophotometry at $480 \mathrm{~nm}$. A profile showing cumulative drug release was plotted as a function of time. Each diffusion experiment was repeated in triplicate.

\section{Photoresponse of NOP-DOX@BSA-FA}

Photoresponsive drug release from NOP-DOX@BSA-FA was studied by its absorption properties. NOP-DOX@BSA-FA pallet $(5 \mathrm{mg}$ ) was irradiated at room temperature using light from a light-emitting diode (LED) array. The corresponding UV-VIS spectra of the pallet were obtained at UV-VIS/NIR (LAMBDA $^{\text {TM }}$ 950; PerkinElmer) after regular time intervals.

\section{Cell Culture}

Human cervical epithelial malignant carcinoma cell lines (HeLa) were maintained in Dulbecco's Modified Eagle's Medium (DMEM) supplemented with 10\% heat inactivated fetal calf serum (Gibco ${ }^{\mathrm{TM}}$, Thermo Fisher Scientific), penicillin $(100 \mathrm{U} / \mathrm{mL})$ and streptomycin $(100 \mu \mathrm{g} / \mathrm{mL})$ at $37^{\circ} \mathrm{C}$ in a humidified atmosphere containing $5 \% \mathrm{CO}_{2}$. HeLa cells at a concentration of $1.5 \times 10^{5}$ cells $/ \mathrm{mL}$ were grown in a $25 \mathrm{~cm}^{2}$ flask of complete culture medium. The study was approved by the Research Ethical Committee of Institute of Biomedical and Genetic Engineering, Islamabad.

\section{MTT assay}

NOPs and their drug loaded conjugates were diluted with DMEM containing $10 \%$ fetal bovine serum (FBS). The cells were incubated with NOPs, NOP-DOX@BSA, and NOP-DOX@BSA-FA for 4 hours in complete darkness. For the nontreated negative control group (NTC), the fresh culture medium without any nanoconjugate was added to the wells. Cells were then exposed to light from the LED array. The rise in temperature was also recorded after every 5 minutes to investigate the involvement of hyperthermia. A Eutech temperature probe (Thermo Fisher Scientific) was used for this purpose. The cells were incubated for 24 hours and cell viability was measured by MTT assay. Noncellular background was subtracted from the respective samples and the percent cell viability (\%) was calculated using the following formula:

$$
\text { Cell viability }(\%)=\frac{\mathrm{OD}_{\text {treated }}}{\mathrm{OD}_{\text {control }}} \times 100
$$

where OD is the optical density. A separate 96-well plate was used for each concentration of NPs and their drug loaded conjugates.

\section{Fluorescence microscopy}

Differential staining of NOP-DOX@BSA-FA $(100 \mu \mathrm{g} / \mathrm{mL})$ treated HeLa cells was done using DNA-intercalating fluorescent dyes acridine orange ( $\mathrm{AO} ; 100 \mu \mathrm{g} / \mathrm{mL})$ and ethidium bromide $(\mathrm{EB} ; 100 \mu \mathrm{g} / \mathrm{mL}$ ). Increase in fluorescence was analyzed under a fluorescent microscope (Leica Microsystems, Wetzlar, Germany) with a Canon camera (Canon Inc., Tokyo, Japan) using 485 and $530 \mathrm{~nm}$ filters for excitation and emission wavelengths, respectively. Nuclear irregularity was measured by ImageJ software (ImageJ 1.42 free version).

To evaluate membrane permeability, NOP-DOX@ BSA-FA treated HeLa cells were stained with $1 \mu \mathrm{M}$ SYTOX green dye for 15 minutes in the dark, and then mounted on a slide. The increase in fluorescence was analyzed as mentioned earlier.

\section{Intracellular ROS generation}

For quenching experiments, HeLa cells were pretreated with sodium azide $\left(\mathrm{NaN}_{3} ; 50 \mathrm{mM}\right)$ and mannitol $(1 \mathrm{mM})$ solution mixed in PBS and incubated for 1 hour, followed by photoirradiation, and subjected to ROS analysis. There were five experimental groups. The first group was incubated with sodium azide $\left(\mathrm{NaN}_{3}\right)$, the second with mannitol, and the third group was incubated with a combination of both $\mathrm{NaN}_{3}$ and mannitol. In the fourth group, cells were exposed to both $\mathrm{NaN}_{3}$ and mannitol without any NPs for any inhibitory effect of these scavengers. In the fifth groups, HeLa cells 
were treated with NPs without any scavenger. Cell viability was measured using the MTT assay as described in the MTT assay section.

\section{Statistical analysis}

All results are expressed as mean \pm standard deviation (SD). Statistical analysis was performed using SPSS 19 software (SPSS, Chicago, IL, USA) and statistical comparisons were made using one-way analysis of variance (ANOVA), followed by Tukey's HSD post-hoc test. The results were considered to be significantly different at a $P$-value $<0.05$.

\section{Results and discussion}

NOPs were synthesized via thermolysis of nickel acetylacetonate (II) $\left(\mathrm{Ni}[\mathrm{acac}]_{2}\right)$ in oleic acid and oleylamine (1:1) acting as solvents. To obtain highly biocompatible and water-soluble dispersion, NOPs were subjected to the ligand exchange process in tetrahydrofuran using sodium citrate and $\mathrm{NaHCO}_{3}$. The composition and crystalline structure of NOPs was confirmed through X-ray diffraction and Rutherford backscattering analysis (Figure S1). The resulting hydrophilic NOPs demonstrated pronounced chemical stability and displayed no evidence of oxidation or degradation, as indicated by the X-ray diffraction monitored for a period of 3 months (Figure S1A). By utilizing the $\%$ reflectance, band gap energies were found to vary from 1.5 to $2.5 \mathrm{eV}$ indicating low-bandgap semiconductor properties (Figure S2). The field emission scanning electron microscopy micrograph showed an approximate particle size of 20-25 nm (Figure 1A). However, using dynamic light

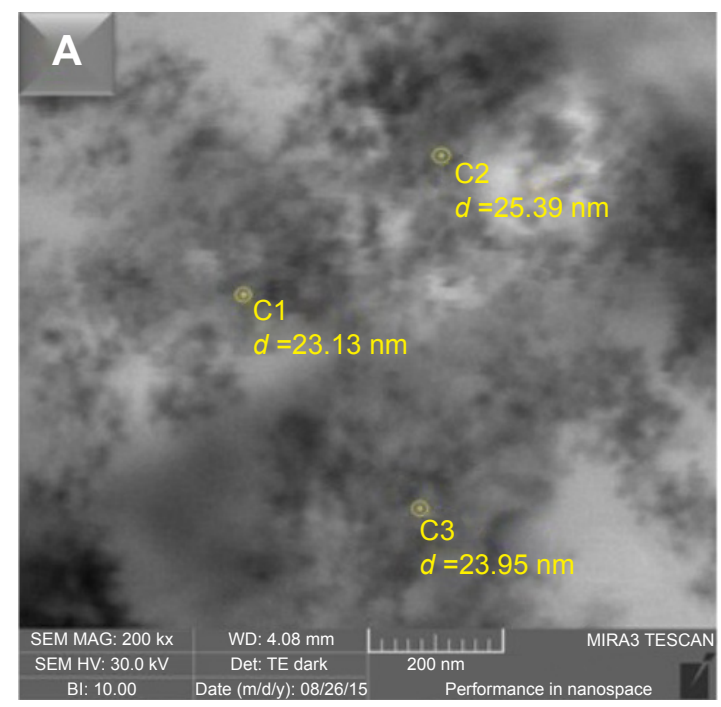

scattering analysis, the average hydrodynamic diameter of NOPs was found to be $29.58 \mathrm{~nm}$ (Figure S3).

We loaded our model anticancer drug DOX on to the surface of water-dispersible NOPs via electronic interactions. The resulting NOP-DOX nanoconjugate was used as core and a BSA shell was constructed through the cross-linking procedure using $6 \%$ glutaraldehyde. BSA was covalently linked with FA through standard carbodiimide chemistry. $\mathrm{NiO}$ nanocrystals were found capable of loading high amount of DOX (3.3 mg/mg of NOPs), whereas BSA shell facilitated in cancer cell targeting and preventing drug leakage during transit to target site. ${ }^{13,14}$ Successful formation of NOP-DOX@ BSA and NOP-DOX@BSA-FA was confirmed through field emission scanning electron microscopy, ultraviolet-visible spectroscopy (UV-VIS), and Fourier transform infrared spectroscopy (Figures 2, $\underline{\mathrm{S} 4 \mathrm{~A}}$ and $\underline{\mathrm{B}}$ ). The electronic interaction of DOX with the NOP surface was confirmed by fluorescence quenching of photoluminescence peak at $652 \mathrm{~nm}$ (Figure S4C).

The aqueous NOP-DOX@BSA-FA suspension showed no aggregation at physiological $\mathrm{pH}$, and was found to be stable even 2 months after their preparation (Figure S4D). Release of DOX from NOP-DOX@BSA-FA was found to be a slow $\mathrm{pH}$-dependent process (Figure 1A). The release studies were performed at $\mathrm{pH}$ 5.4, 7.4, and 9 in phosphate-buffered saline. The NOP-DOX@BSA-FA was dialyzed for 64 hours under normal conditions and revealed that the release of DOX was approximately twofold greater at $\mathrm{pH} 5.4$ than physiological $\mathrm{pH}$ (7.4). The rapid DOX release rates at lower $\mathrm{pH}$ were

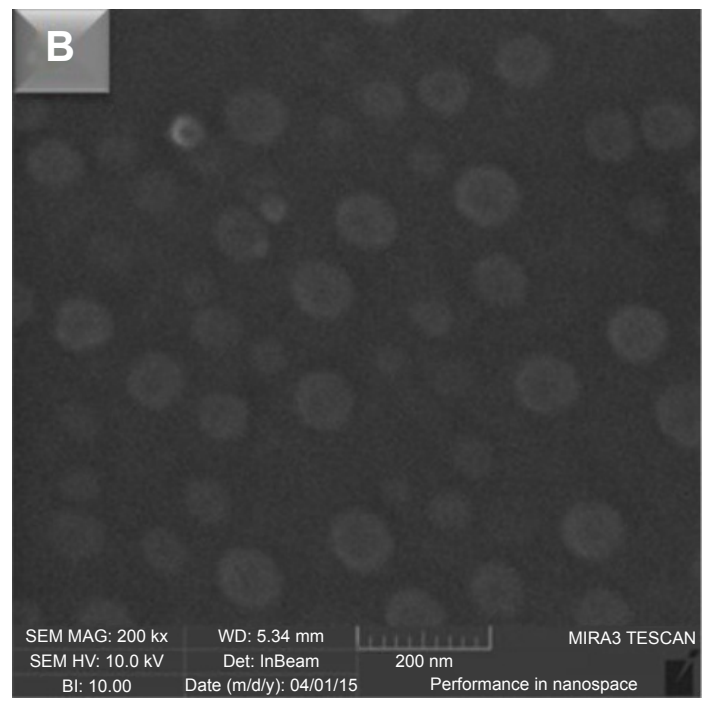

Figure I FE-SEM images of NOPs and NOP-DOX@BSA nanospheres.

Notes: (A) Image of $\mathrm{NiO}$ obtained from FE-SEM instrument with scanning transmission electron microscope detector showing the size distribution of nanoparticles in the range of 20-25 nm. (B) FE-SEM images of NOP-DOX@BSA-FA nanospheres showing somewhat spherical shape and increased size of up to $80 \mathrm{~nm}$.

Abbreviations: BSA, bovine serum albumin; DOX, doxorubicin; FA, folic acid; FE-SEM, field emission scanning electron microscopy; NOPs, nickel oxide nanoparticles. 


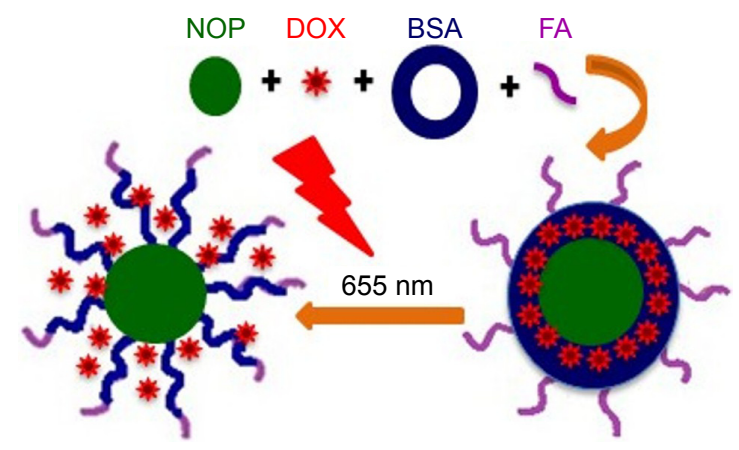

Figure 2 The scheme of this work.

Abbreviations: BSA, bovine serum albumin; DOX, doxorubicin; FA, folic acid; NOP, nickel oxide nanoparticle.

very impressive for targeting tumors, as the tumor microenvironment and intracellular drug-trafficking vehicles such as liposomes had acidic pH (Figure 3A).

Although the photoactive response of BSA is already documented in the literature, ${ }^{15}$ the photoactive response of BSA conjugates is not yet reported. Keeping in view the absorption by NOPs, photoresponsive drug release has been studied exposing $5 \mathrm{mg}$ pellet of NOP-DOX@BSA-FA to light from a LED panel $\left(\lambda_{\max }=655 \mathrm{~nm} ; 50 \mathrm{~mW} / \mathrm{cm}^{2}\right)$. Upon exposure to red light, almost $70 \%$ of DOX was released just in 30 minutes. The results clearly indicated a robust DOX release leading to $\sim 100$-fold increase in release rate (Figure 3B).

The impact of red light exposure upon NOP-DOX@ BSA-FA was clearly manifested through the UV-VIS absorption spectra collected as a function of time (Figure S5A and B). Prior to irradiation, NOP-DOX@ BSA-FA showed absorption features at longer wavelengths (at $t=0$ top curve, Figure S5B) that resulted from a weighted contribution of FA ( $\lambda_{\text {max }}=280 \mathrm{~nm}, \varepsilon=25,545 \mathrm{M}^{-1} \mathrm{~cm}^{-1} ; 364 \mathrm{~nm}$, $\left.\varepsilon=6,676 \mathrm{M}^{-1} \mathrm{~cm}^{-1}\right), \operatorname{DOX}\left(\lambda_{\max }=480 \mathrm{~nm}, \varepsilon=17,376 \mathrm{M}^{-1} \mathrm{~cm}^{-1}\right)$, and BSA $\left(\lambda_{\max }=277 \mathrm{~nm}, \varepsilon=43,824 \mathrm{M}^{-1} \mathrm{~cm}^{-1}\right)$. A large decrease in absorbance at $270 \mathrm{~nm}$ was observed with $\sim 70 \%$ of change in 30 minutes. There was a negligible shift in absorption maxima at different density of radiations. This spectral feature may be attributed to the structural and conformational changes in BSA after getting energy from the excited NOPs (Figure S5A). This leads to the light-sensitive release characteristics in NOP-DOX@BSA-FA conjugate.

The potential of NOPs for light-triggered DOX delivery together with the photodynamic cancer treatment was determined through the cell viability. By varying the treatment parameters, it was found that 30 minutes of irradiation from a LED panel resulted in $90 \%$ inhibition in case of NOP-DOX@BSA-FA and 50\% in case of NOPs. Figure 3C shows inhibited cell growth as a function of exposure time. While DOX was used in the same amount $(5.7 \mathrm{nM})$, the cellular deaths were found to be more than eightfold higher for NOPs-DOX@BSA-FA compared to free DOX. A very high cellular death rate encountered in NOP-DOX@ BSA-FA under light exposure most probably originated due to the cumulative effect of enhanced drug release from NOPs-DOX@BSA-FA construct and PDT effect of NOPs (Figure 3D). However, NOP-DOX@BSA-FA has already shown photoresponsive drug release under irradiation from a LED panel (Figure 3B). A significant in vitro cell death estimation was followed by fluorescence microscopy of HeLa cells treated with NOPs. Figure 4 shows the appearance of HeLa cells stained with apoptotic dyes acridine orange and ethidium bromide. ${ }^{16}$ Microscopic visualization revealed the emission of respective green and red colors. The density of HeLa cells, emitting green fluorescence, was highest for 5 minutes of light exposure. Almost $50 \%$ of cell death was found at 15 minutes of light exposure. Characteristic features of apoptotic cells, such as cytoplasmic membrane blebbing, nuclear contraction, nuclear fragmentation, and contact inhibition, were visible at 30 minutes exposure (showing orange fluorescence due to acridine orange stain).

The apoptotic cell death in HeLa could be a result of the PDT effect of NOPs. To verify, we first tested the ROS generation potential of NOPs under red light exposure. Generation of ${ }^{1} \mathrm{O}_{2}$ by photo excited NOPs in ethanol was detected by 1,3-diphenylisobenzofuran. Singlet oxygen quantum yield $\Phi_{\text {NOP }}$ turned out to be $0.64 \pm 0.02$, which is $\sim 23 \%$ higher than methylene blue $\left(\Phi_{\mathrm{MB}}=0.52 \pm 0.03\right)$. To further confirm the PDT-assisted cell killing, MTT assay was performed with and without preincubation of HeLa cells with sodium azide and mannitol. As shown in Figure 5A, cell death by NOPs, NOP-DOX@BSA, and DOX@BSA-FA without light irradiation is far less than under light irradiation. Moreover, pretreatment of HeLa cells by sodium azide vividly overturned cell death, indicating that ${ }^{1} \mathrm{O}_{2}$ was the major ROS produced from NOPs. The results shown in Figure 5A unambiguously support the argument that the cellular death upon photoirradiation was mostly due to the PDT effect. Moreover, only a negligible increase $\left(2.8^{\circ} \mathrm{C} \pm 0.32^{\circ} \mathrm{C}\right)$ in cell culture temperature after light exposure was observed, which eliminated the involvement of hyperthermia in photokilling of HeLa cells. Thiobarbituric acid reactive substances assay was also performed on HeLa cells under light and dark conditions (Figure 5B). Both NOPs and NOP-DOX@BSA were found to significantly enhance the thiobarbituric acid 
A
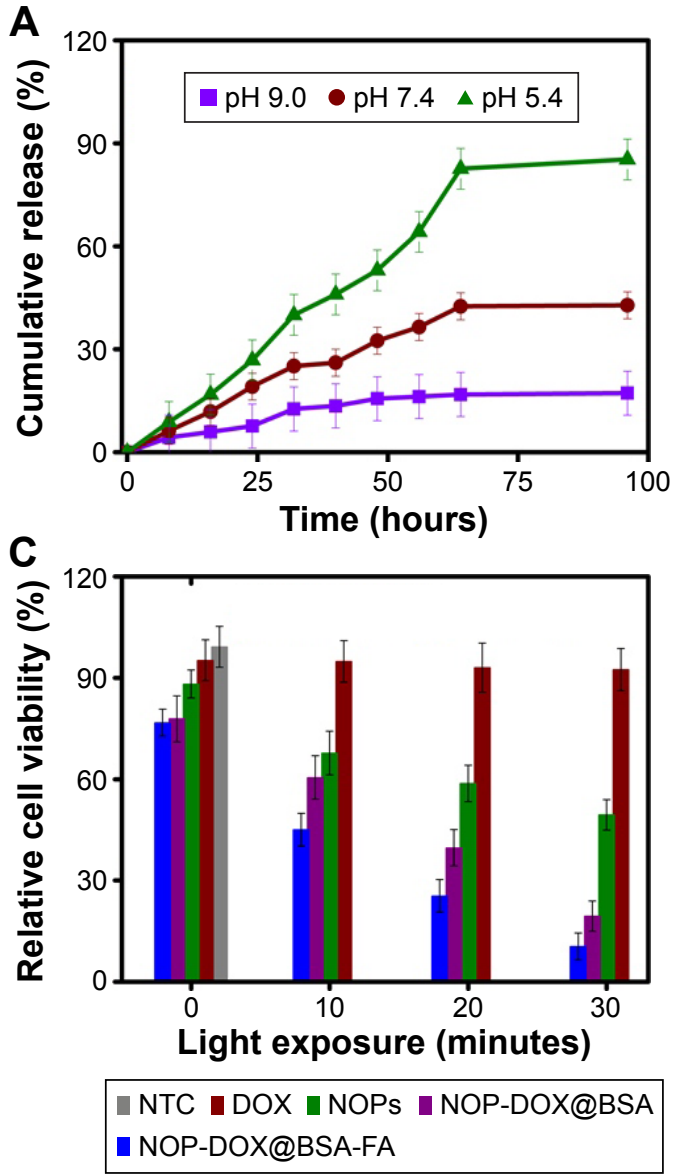

B

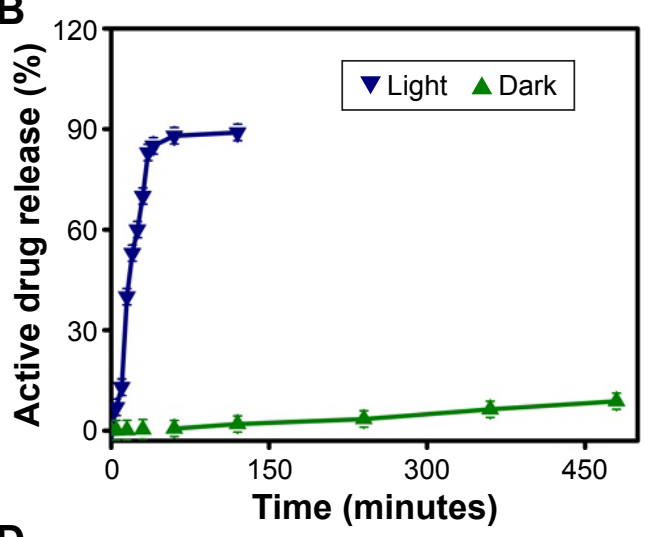

D



Figure 3 Drug release, cell viability, and PDT affect studies.

Notes: (A) Time-dependent drug release with varying $\mathrm{pH}$ under normal laboratory condition. (B) Comparison of drug release studies for a period of 480 minutes with light source and in complete darkness from NOP-DOX@BSA-FA. No significant drug release was observed in the first 30 minutes under dark conditions at pH 5.4 , while $>70 \%$ drug was released under light. (C) Relative cell viability studies as a function of irradiation time. Light exposure had no effect on the viability of NTC, very negligible effect on DOX-treated cells, whereas it had significant effect on NOPs, NOP-DOX@BSA-, and NOP-DOX@BSA-FA-treated cells. (D) The ratio of \%CV with light irradiation to that without light irradiation ( $\mathrm{CV}+/ \mathrm{CV}-)$.

Abbreviations: BSA, bovine serum albumin; CV, cell viability; DOX, doxorubicin; FA, folic acid; NOPs, nickel oxide nanoparticles; NTC, nontreated cells; PDT, photodynamic treatment.
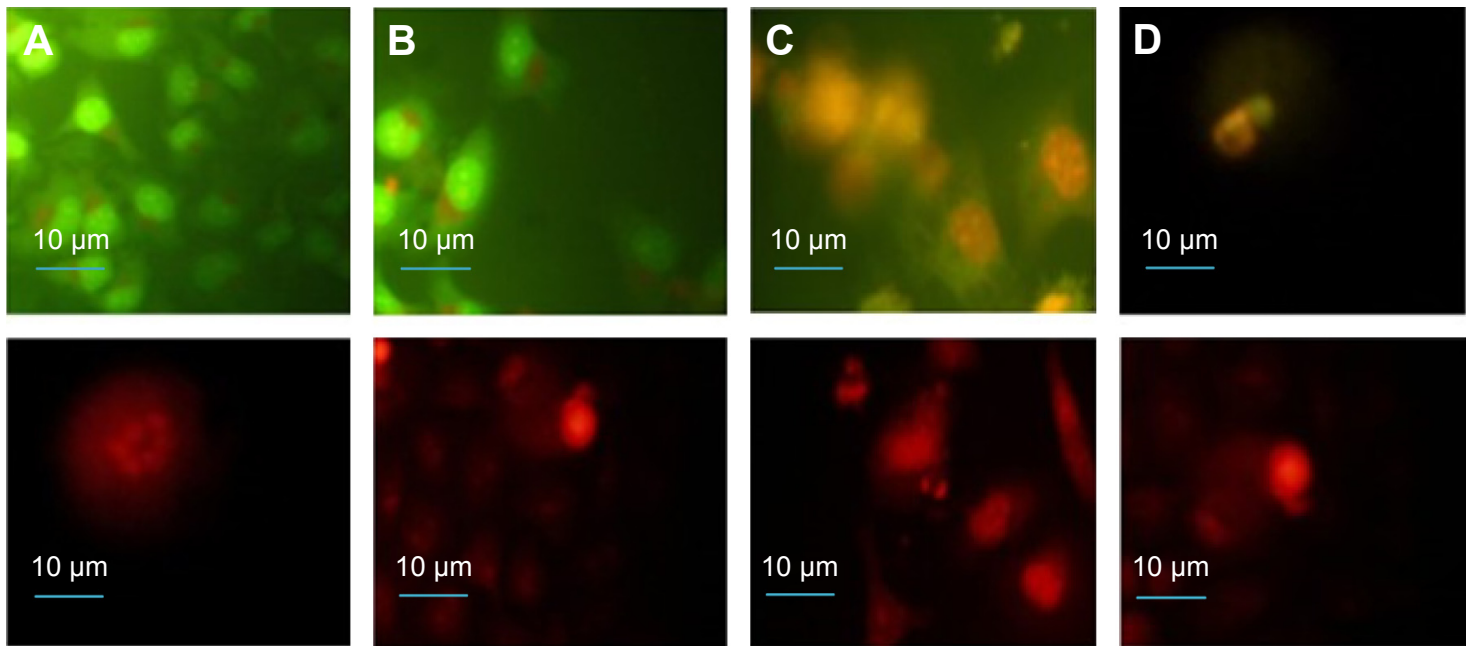

Figure 4 Fluorescence microcopy images of HeLa cells stained with AO (first row) and EB (second row) after exposure to NOP-DOX@BSA-FA and a light dose. Notes: (A) Cells exposed for 5 minutes showing the formation of apoptotic bodies within the cells; (B) apoptotic and necrotic cells at I5 minutes, with $50 \%$ of the cells showing necrosis and the remaining showing early and late apoptosis; and (C, D) apoptotic and necrotic cells at 30 minutes exposure showing nuclear condensation, budding to form apoptotic bodies, and nuclear fragmentation. Magnification is $60 X$.

Abbreviations: AO, acridine orange; BSA, bovine serum albumin; DOX, doxorubicin; EB, ethidium bromide; FA, folic acid; HeLa, human cervical epithelial malignant carcinoma; NOP, nickel oxide nanoparticle. 
A

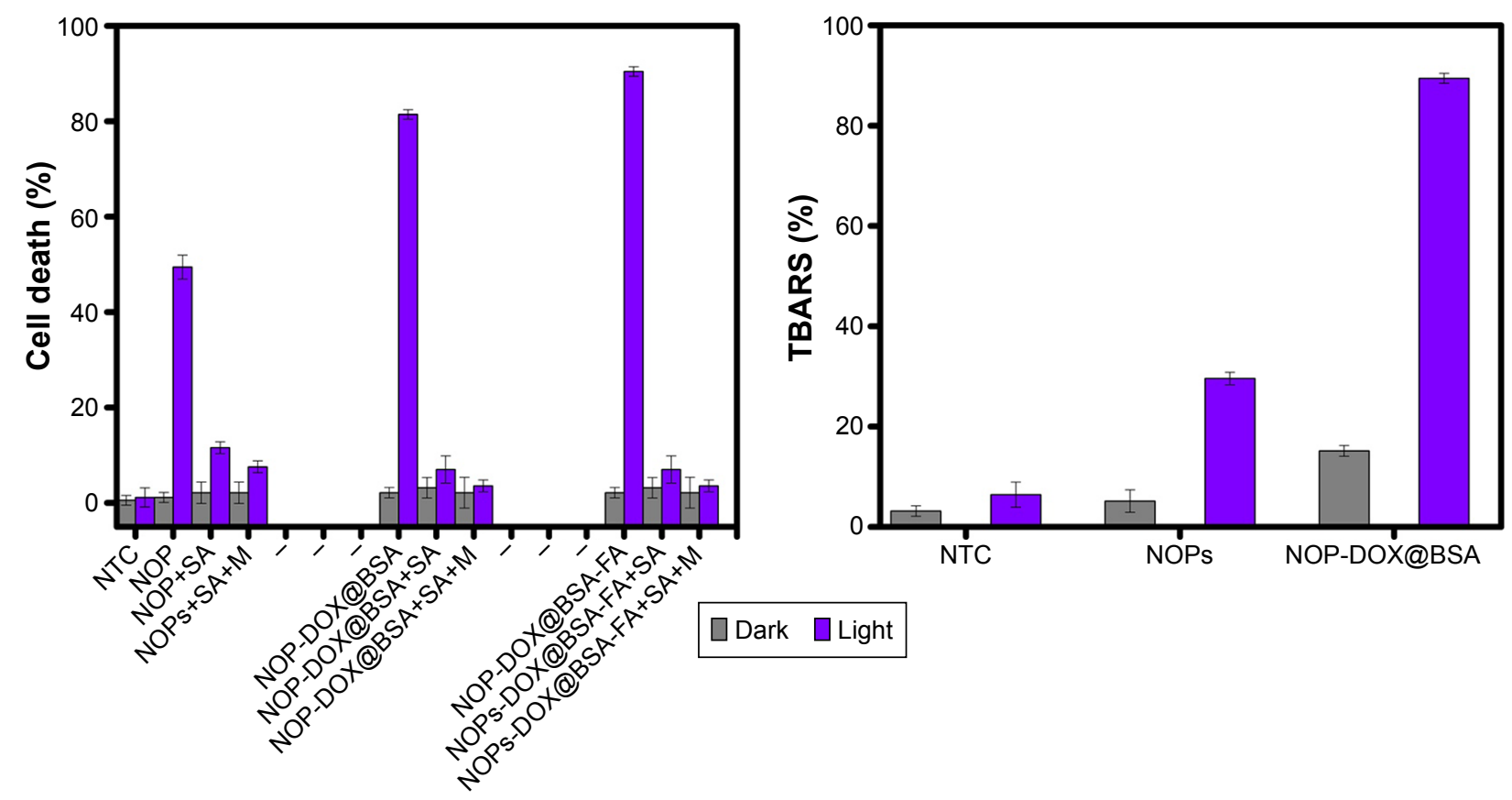

Figure 5 Effect of ROS scavenger and TBA.

Notes: (A) Effect of ROS scavenger M and SA on the photo-oxidative activity of NOPs, NOP-DOX@BSA, and NOP-DOX@BSA-FA. (B) TBA assay results for NOPs and NOP-DOX@BSA exposure. Data are expressed as percent (\%) TBARS. Values represent mean \pm SD. $P<0.000$ I when compared to dark and light.

Abbreviations: BSA, bovine serum albumin; DOX, doxorubicin; FA, folic acid; M, mannitol; NOP, nickel oxide nanoparticle; NTC, nontreated cells; ROS, reactive oxygen species; SA, sodium azide; SD, standard deviation; TBARS, thiobarbituric acid reactive substances; TBA, thiobarbituric acid.

reactive substances levels on exposure to light, confirming the photodynamic killing of HeLa cells.

\section{Conclusion}

We reported a unique NOP-DOX@BSA system for combined drug delivery and PDT. Our drug carrier system is adept at red light-triggered drug release, giving $\sim 100 \%$ release enhancement compared to the dark conditions besides a desired $\mathrm{pH}$-sensitive release for tumor targeting. Light exposure was found to enhance DOX delivery; however, cellular deaths mostly originated due to PDT effect, making NOP-DOX@BSA a prevailing system for PDT and light trigger-based targeted drug delivery. Red light exposure did not affect nontreated cells and slightly affected cells incubated with free DOX. "Smart" NOPs could offer new avenues for effective drug delivery of chemotherapeutic or imaging agents, allowing PDT while reducing drug toxicity to systemic and nontargeted tissues.

\section{Acknowledgments}

The authors acknowledge the generous fund of deanship of research in King Saud University, Kingdom of Saudi Arabia, under research group grant no RG-150. The authors are grateful to Mr Abdul Haleem, PhD student, Department of Microbiology, Quaid-i-Azam University, and Mr Naeem at National Centre for Physics for UV-VIS and Fourier transform infrared spectroscopy instrumentation time and assistance. Ms Shazia Bano gives thanks to the Higher Education Commission (HEC) for the Indigenous Fellowship for their support regarding her PhD studies.

\section{Disclosure}

The authors report no conflicts of interest in this work.

\section{References}

1. Ojima I, Zuniga ES, Berger WT, Seitz JD. Tumor-targeting drug delivery of new-generation taxoids. Future Med Chem. 2012;4(1):33-50.

2. Abbasi E, Aval SF, Akbarzadeh A, et al. Dendrimers: synthesis, applications, and properties. Nanoscale Res Lett. 2014;9(1):247.

3. Lim E-K, Jang E, Lee K, Haam S, Huh Y-M. Delivery of cancer therapeutics using nanotechnology. Pharmaceutics. 2013;5(2):294-317.

4. Allen TM, Cullis PR. Liposomal drug delivery systems: From concept to clinical applications. Adv Drug Deliv Rev. 2012;65(1):36-48.

5. Liechty WB, Kryscio DR, Slaughter BV, Peppas NA. Polymers for drug delivery systems. Annu Rev Chem Biomol Eng. 2012;1:149-173.

6. Adeyemi OS, Sulaiman FA. Evaluation of metal nanoparticles for drug delivery systems. J Biomed Res. 2015;29(2):145-149.

7. Vandecruys E, Mondelaers V, De Wolf D, Benoit Y, Suys B. Late cardiotoxicity after low dose of anthracycline therapy for acute lymphoblastic leukemia in childhood. J Cancer Surviv. 2012;6(1):95-101.

8. Liu X, Fu F, Xu K, et al. Folic acid-conjugated hollow mesoporous silica/CuS nanocomposites as a difunctional nanoplatform for targeted chemo-photothermal therapy of cancer cells. J Mater Chem B. 2014; 2(33):5358-5367. 
9. Lv R, Yang P, He F, et al. A yolk-like multifunctional platform for multimodal imaging and synergistic therapy triggered by a single nearinfrared light. ACS Nano. 2015;9(2):1630-1647.

10. Fang W, Yang J, Gong J, Zheng N. Photo- and pH-triggered release of anticancer drugs from mesoporous silica-coated Pd@Ag nanoparticles. Adv Funct Mater. 2011;22(4):842-848.

11. Wang Y, Zhao R, Wang S, Liu Z, Tang R. In vivo dual-targeted chemotherapy of drug resistant cancer by rationally designed nanocarrier. Biomaterials. 2016;75:71-81.

12. Xiao L, Gu L, Howell SB, Sailor MJ. Porous silicon nanoparticle photosensitizers for singlet oxygen and their phototoxicity against cancer cells. ACS Nano. 2011;5(5):3651-3659.

13. Zu Y, Zhang Y, Zhao X, Zhang Q, Liu Y, Jiang R. Optimization of the preparation process of vinblastine sulfate (VBLS)-loaded folateconjugated bovine serum albumin (BSA) nanoparticles for tumortargeted drug delivery using response surface methodology (RSM). Int J Nanomedicine. 2009;4:321-333.
14. Wu Y, Ihme S, Feuring-Buske M, et al. A core-shell albumin copolymer nanotransporter for high capacity loading and two-step release of doxorubicin with enhanced anti-leukemia activity. Adv Healthc Mater. 2013;2(6):884-894.

15. Gnanadhas DP, Ben Thomas M, Thomas R, Raichur AM, Chakravortty D. Interaction of silver nanoparticles with serum proteins affects their antimicrobial activity in vivo. Antimicrob Agents Chemother. 2013; 57(10):4945-4955

16. Ribble D, Goldstein NB, Norris DA, Shellman YG. A simple technique for quantifying apoptosis in 96-well plates. BMC Biotechnol. 2005;5:12.

\section{Publish your work in this journal}

The International Journal of Nanomedicine is an international, peerreviewed journal focusing on the application of nanotechnology in diagnostics, therapeutics, and drug delivery systems throughout the biomedical field. This journal is indexed on PubMed Central, MedLine, CAS, SciSearch $\AA$, Current Contents ${ }^{\circledR} /$ Clinical Medicine,
Journal Citation Reports/Science Edition, EMBase, Scopus and the Elsevier Bibliographic databases. The manuscript management system is completely online and includes a very quick and fair peer-review system, which is all easy to use. Visit http://www.dovepress.com/ testimonials.php to read real quotes from published authors. 